
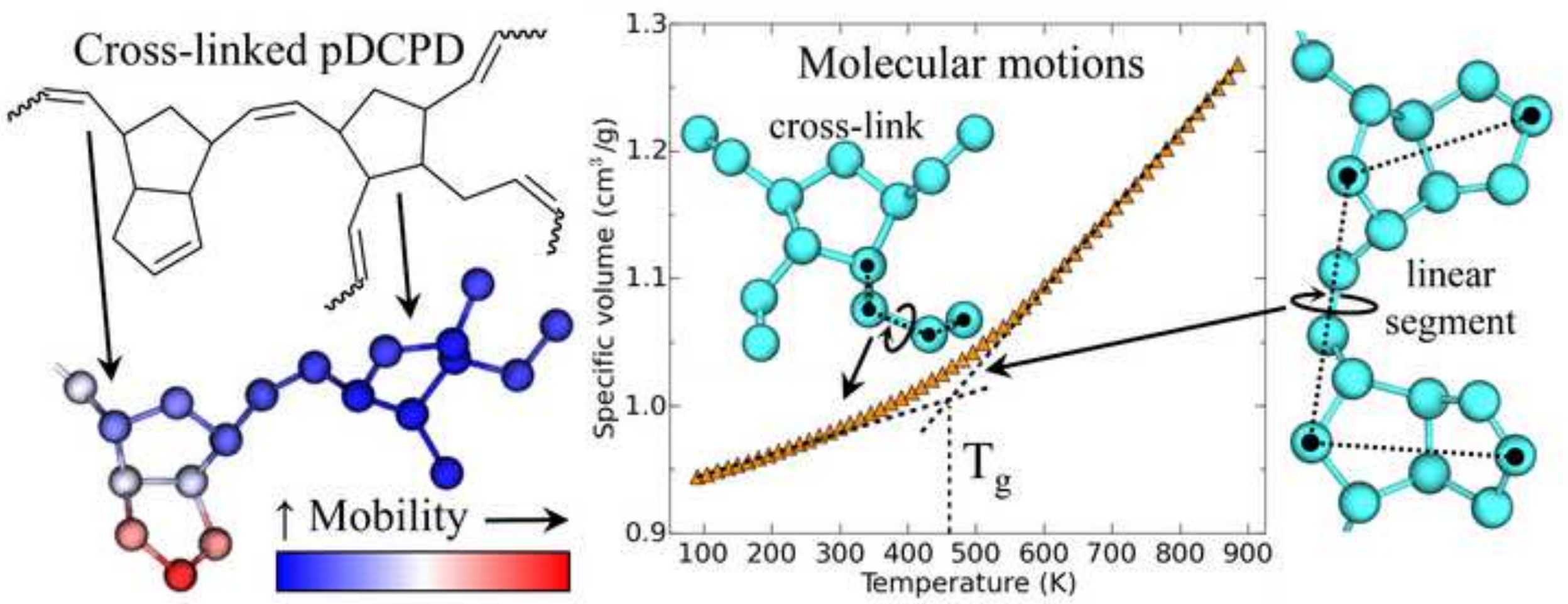


\title{
A molecular simulation study of the glass transition of cross-linked poly(dicyclopentadiene) networks
}

\author{
Robert M. Elder, Jan W. Andzelm, Timothy W. Sirk* \\ U.S. Army Research Laboratory, Aberdeen Proving Ground, Maryland 21005, USA
}

* corresponding author: Timothy W. Sirk, timothy.w.sirk.civ@ mail.mil 


\section{Introduction}

Cross-linked polymer networks are widely used in commercial and military applications, where they function as structural and protective materials [1-3]. In particular, the resistance of cross-linked polymers to high strain rate impact events is desirable for many applications. Accordingly, numerous experimental studies of cross-linked polymers, such as epoxy resins, have been conducted with the overall goal of improving impact resistance [1,4-6]. Although epoxy resins are widely used in applications requiring ballistic performance, resins with the high strength and stiffness necessary for structural applications typically have inferior toughness [7]. In a recent study, we demonstrated that alternative chemistries can overcome this trade-off between strength and toughness [8]. Specifically, cross-linked poly(dicyclopentadiene) (pDCPD) has exceptional ballistic performance compared to epoxy resins, which appears to be related to the high fracture toughness of pDCPD. The molecular mechanism underlying this exceptional toughness was not readily identifiable, but it is desirable to determine this mechanism.

Distinctively, pDCPD is a pure hydrocarbon (Fig. 1), therefore causing the chemical structure and physical properties to differ sharply from typical epoxy-amine polymer networks. One chemistrydependent contribution to mechanical properties are molecular motions at the glass transition and in the glassy state, i.e. sub- $\mathrm{T}_{\mathrm{g}}$ relaxations $[9,10]$. These motions have been reported to improve mechanical properties by dissipating energy $[9,10]$ and are well-understood in epoxies and other common network chemistries [11]. But, despite the common use of pDCPD in applications requiring stiffness, impact resistance, and corrosion resistance, such as chemical process equipment and coverings for heavy machinery, its molecular-level behavior is not well-studied $[4,8,12,13]$. In this work, we analyze the molecular relaxation mechanisms of pDCPD networks which underlie the experimental observations of temperature- and frequency-dependent bulk properties. 

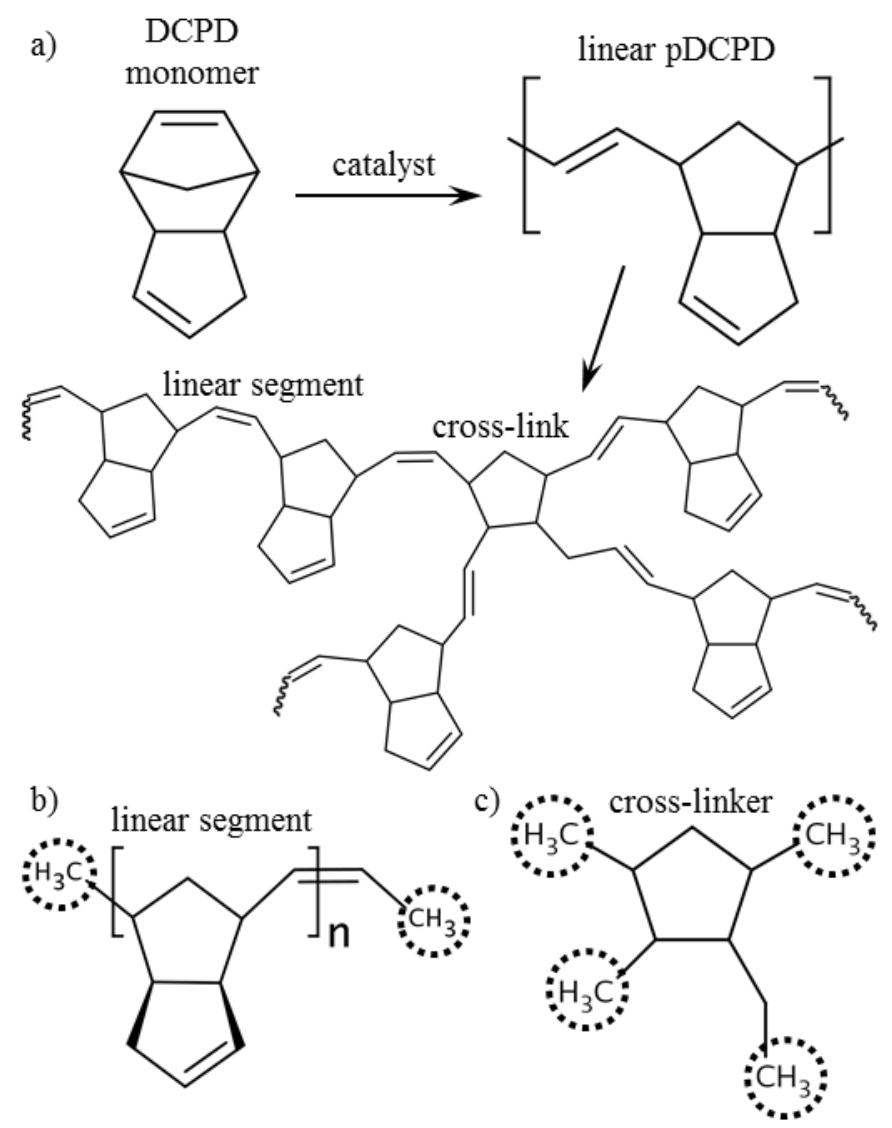

Figure 1. a) Schematic of the reaction mechanism for catalyzed ring-opening metathesis polymerization (ROMP) of dicyclopentadiene (DCPD) into a cross-linked polymer network. b) The linear segment used to construct simulated pDCPD networks. The length or degree of polymerization of the linear segment, $n$, varies. Circles indicate the carbon atoms between which cross-linking bonds were formed during simulated polymerization. c) The cross-linker used to construct simulated pDCPD networks.

\section{Methods}

Design of pDCPD networks for simulations. Motivated by a recent experimental study of pDCPD networks [8], we constructed cross-linked pDCPD networks based on the chemical structure shown in Fig. 1a. Because the molecular weight between cross-links, $\mathbf{M}_{\mathrm{C}}$, strongly affects the properties of cross-linked polymers [14], we constructed models with $\mathrm{M}_{\mathrm{C}}$ similar to the pDCPD network synthesized by Knorr et al. [8]. Although $\mathrm{M}_{\mathrm{C}}$ can be measured experimentally with dynamic mechanical analysis (DMA) and the theory of rubber elasticity, uncertainty is introduced by finite chain stiffness and interchain interactions [15]. Therefore, we constructed three pDCPD networks with different $\mathrm{M}_{\mathrm{C}}$ by using linear segments composed of $n$ monomers (Fig. 1b) between cross-linkers (Fig. 1c). Two of the pDCPD networks, pDCPD-3 $\left(n=3, \mathrm{M}_{\mathrm{C}}=463 \mathrm{~g} / \mathrm{mol}\right)$ and pDCPD-6 $\left(n=6, \mathrm{M}_{\mathrm{C}}=859 \mathrm{~g} / \mathrm{mol}\right)$ encompass the experimental $\mathrm{M}_{\mathrm{C}}$ value $(670 \mathrm{~g} / \mathrm{mol},[8])$ and provide information about the variation of pDCPD network 

about the behavior of highly cross-linked pDCPD networks. We calculate $\mathrm{M}_{\mathrm{C}}$ from the network stoichiometry as suggested by Crawford and Lesser [15] as

$$
M_{c}=2\left(\frac{M W_{L}}{2}+\frac{M W_{X}}{f}\right)
$$

where $\mathrm{MW}_{\mathrm{L}}$ is the molecular weight of the linear segment, $\mathrm{MW}_{\mathrm{X}}$ is the molecular weight of the crosslinker, and $f$ is the functionality of the cross-linkers ( $f=4$ in this study).

Besides $\mathrm{M}_{\mathrm{C}}$, several other parameters define a pDCPD network. First, the catalyst or reaction conditions of polymerization may affect the type of cross-linker [16,17]. In this study, we chose the crosslinker structure in which both the cyclopentenic and norbornenic rings have undergone ring-opening [18]. Second, different catalysts or reaction conditions may produce different proportions of cis and trans double bonds within the linear pDCPD segments [16]. For this study, we constructed networks with these double bonds in a cis configuration. Lastly, DCPD typically exists as a mixture of endo and exo isomers. Since commercial mixtures primarily contain endo isomers [19], we constructed pDCPD networks from endo monomers. Future work could examine the effects of alternate cross-linker structures, trans bonds, and exo isomers. Additional details about these systems are given in Table 1. As in previous work [20,21], the relatively large size of these systems ( 200,000 atoms) was chosen to reduce statistical uncertainty [22].

Table 1. Details of pDCPD network systems. The number of molecules (\#), number of atoms $(N)$, length of linear segments $(n)$, and molecular weight between cross-links $\left(\mathrm{M}_{\mathrm{C}}\right)$ are shown for each network.

\begin{tabular}{cccccc}
\hline $\begin{array}{c}\text { Polymer } \\
\text { network }\end{array}$ & $\begin{array}{c}\text { \# linear } \\
\text { molecules }\end{array}$ & $\begin{array}{c}\text { \# cross-linker } \\
\text { molecules }\end{array}$ & $\boldsymbol{N}$ & $\boldsymbol{n}$ & $\begin{array}{c}\mathbf{M}_{\mathbf{C}} \\
(\mathbf{g} / \mathbf{m o l})\end{array}$ \\
\hline pDCPD-1 & 6300 & 3150 & 207900 & 1 & 198 \\
pDCPD-3 & 2800 & 1400 & 215600 & 3 & 463 \\
pDCPD-6 & 1458 & 729 & 208494 & 6 & 859 \\
\hline
\end{tabular}

Construction of cross-linked polymer networks. The cross-linked networks used in this study were constructed using Monte Carlo simulated annealing [23,24], which we have previously used to construct cross-linked epoxy networks $[8,20,21,24]$. Briefly, the steps of this procedure are as follows. A stoichiometric reaction mixture of linear segments and cross-linkers (Fig. 1b and Fig. 1c) is equilibrated using constant NPT MD simulation at $735 \mathrm{~K}$. Next, the network topology (i.e., the connectivity of linear segments and cross-linkers) is assigned using the simulated annealing algorithm, which minimizes the total distance between all pairs of reacting atoms. For pDCPD, the reacting atoms are the carbon atoms in the methyl groups indicated in Fig. 1b and Fig. 1c. The identified bonds are then created and gradually relaxed to their equilibrium bond lengths in a series of short MD simulations; hydrogen atoms that are eliminated as products of the cross-linking reaction (i.e., two hydrogen atoms per reacting methyl group) 
are removed; new angle and dihedral interactions resulting from the creation of cross-linking bonds are added; and the partial charges of atoms within two bonds of the cross-linking atoms are adjusted. Finally, the structure is relaxed with constant $N P T$ MD simulation at $735 \mathrm{~K}$ for $5 \mathrm{~ns}$. The structures were approximately cubic in shape with side lengths of about $13 \mathrm{~nm}$ at $735 \mathrm{~K}$. To reduce statistical uncertainty, five independent replicas of each system were prepared by generating different initial velocities for the reaction mixture. Therefore, the network topology of each replica is different.

Characterization of network topology. Network connectivity, or topology, can be represented using mathematical graph structures. In the present study, during the Monte Carlo simulated annealing procedure the only control we exerted on the network graph was to prevent cross-linkers from forming two bonds with a single linear segment (i.e., a one-molecule loop). We initially constructed a pDCPD-3 network where some of the linear segments formed a one-molecule loop. We calculated the per-atom potential energy of atoms in these linear segments [25]. The total potential energy of linear segments in one-molecule loops was approximately $8 \mathrm{~kJ} / \mathrm{mol}$ higher per segment than linear segments not involved in one-molecule loops, which suggests that the formation of these cycles is energetically disfavored during polymerization. Consequently, we chose to exclude one-molecule loops, and therefore the networks constructed here are "ideal" in that they have no short loops that weaken the material by creating elastically inactive chains [26].

The glass transition. The thermal and volumetric properties of the cross-linked polymer networks were assessed by annealing, i.e. a stepwise temperature change of the structures with MD simulations. Starting from a temperature well-above the glass transition $(735 \mathrm{~K})$, the temperature was raised or lowered in steps of $15 \mathrm{~K}$, and a 2 ns constant $N P T$ MD simulation was performed at each new temperature, corresponding to a cooling rate of $7.5 \times 10^{9} \mathrm{~K} \mathrm{~s}^{-1}$. This stepwise heating/cooling procedure was repeated until the range of temperatures spanned from the rubbery state at $885 \mathrm{~K}$ to deep in the glassy state at $90 \mathrm{~K}$. Data from the first $1 \mathrm{~ns}$ of each temperature step was discarded as equilibration time, and the final $1 \mathrm{~ns}$ of each step was used to calculate thermal and volumetric properties.

Validation of network structures. The system properties were examined to verify that the structures were well-equilibrated at high temperature and stable at temperatures below the glass transition. Consistent with our previous work $[20,21,24]$ the volume and potential energy reached a plateau during the initial $5 \mathrm{~ns}$ relaxation at $735 \mathrm{~K}$ and during each subsequent $2 \mathrm{~ns}$ annealing step. Further, we examined the newly formed cross-linking bonds to check for unrealistically extended bonds as a result of the singlestep cross-linking procedure. The average length of the newly formed cross-linking bonds during the $5 \mathrm{~ns}$ relaxation period was found to be essentially equal (within $0.8 \%$ ) to the equilibrium bond length. Thus, we conclude that the networks do not have highly unfavorable bonds as a result of the assigned network topology or other factors. 
Details of simulation protocol. Similar to our previous studies of cross-linked epoxy networks [20,21,24], the general Amber force field [27,28] was applied. Short-range non-bonded interactions were cut off at $9 \AA$, and long-range contributions of van der Waals interactions to the energy and pressure were estimated using tail corrections [29]. Atomic partial charges were calculated using the AM1-BCC method [30,31]. Electrostatic interactions were calculated using the particle-particle particle-mesh (PPPM) method [32]. The velocity-Verlet integrator was used with a time step of 1.0 fs. The simulations were conducted in the NPT ensemble (i.e., constant number of particles, constant pressure, and constant temperature). A Nosé-Hoover thermostat and barostat [33] were used to control temperature and pressure using damping constants of 0.1 and $1.0 \mathrm{ps,} \mathrm{respectively.} \mathrm{Anisotropic} \mathrm{pressure} \mathrm{control} \mathrm{was} \mathrm{used} \mathrm{to}$ maintain a pressure of $1 \mathrm{~atm}$. The simulation box was orthorhombic and periodic in three dimensions. We employed the LAMMPS simulation package [34] (http://lammps.sandia.gov) for all simulations. VMD [35] was used for visualization and analysis. The moltemplate program [36] (http://www.moltemplate.org) was used in constructing the cross-linked polymer networks. 


\section{Results and Discussion}
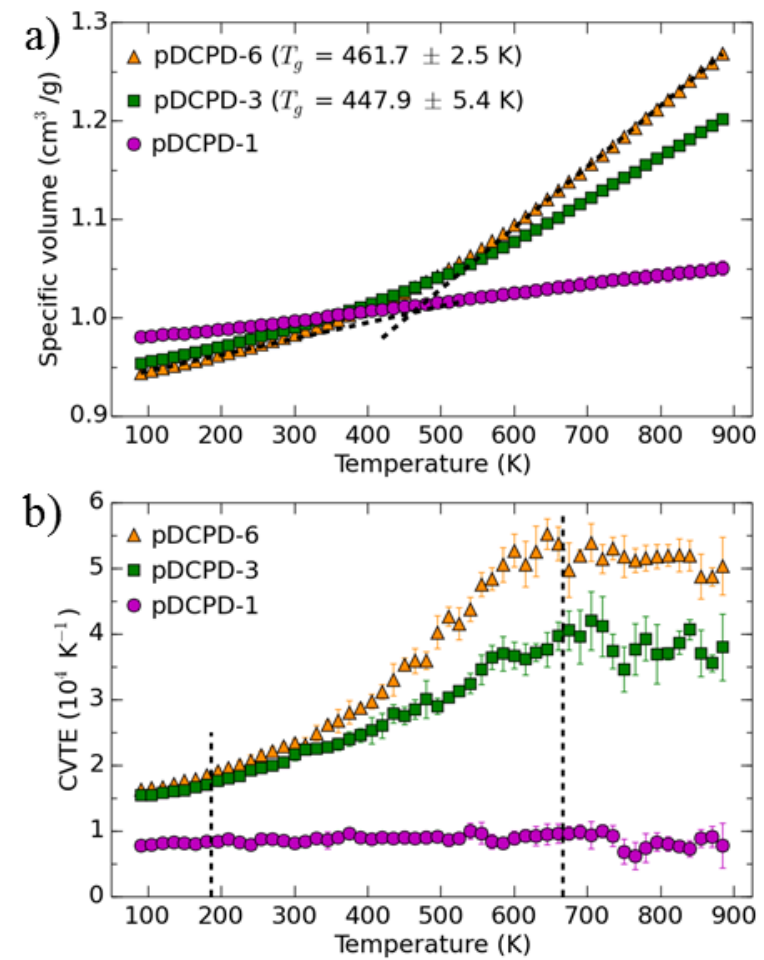

Figure 2. a) Specific volume and b) coefficient of volumetric thermal expansion (CVTE) of pDCPD systems with varying molecular weight between cross-links. Dashed lines in a) indicate the linear fits used to determine the glass transition temperature, $\mathrm{T}_{\mathrm{g}}$, for pDCPD-6; for clarity, linear fits are not shown for the other networks. Dashed vertical lines in b) indicate the temperature ranges where the CVTE is approximately constant ( $\leq 180 \mathrm{~K}$ and $\geq 675 \mathrm{~K})$ and where linear fits were taken to determine $\mathrm{T}_{\mathrm{g}}$. The glass transition temperature of $\mathrm{pDCPD}-1$ could not be determined. Error bars are the standard deviation of five replicas.

\section{A. Volumetric and thermal properties of pDCPD networks.}

During the simulated annealing procedure, we calculated thermal and volumetric properties for the three pDCPD networks. Fig. 2a shows the temperature-dependent specific volume, $v(\mathrm{~T})$, from which the glassy and rubbery regions can be identified as linear trends in $v(\mathrm{~T})$. To identify these linear regions, we calculated the coefficient of volumetric thermal expansion (CVTE):

$$
C V T E=\left(\frac{1}{v} \frac{\partial v}{\partial T}\right)_{P}
$$

As temperature decreases, the CVTE displays three general regions (Fig. 2b). At high temperatures, the CVTE is large and essentially constant. At intermediate temperatures, the CVTE sharply decreases with decreasing temperature. At low temperatures, the CVTE is small and essentially constant. We refer to 
these high and low temperature regions, which are indicated by the dashed vertical lines in Fig. $2 \mathrm{~b}$, as the rubbery and glassy regions, respectively.

We take the glass transition temperature, $\mathrm{T}_{\mathrm{g}}$, as the inflection point in $v(\mathrm{~T})$ between the glassy and rubbery regions. In experiments the glass transition is sharp and well-defined, but in simulations the glass transition is broadened by the high cooling rates that must be used [20]. To determine $T_{g}$ from the simulated $v(\mathrm{~T})$ data, we calculated the intersection of linear fits to the rubbery and glassy regions. The calculated value of $\mathrm{T}_{\mathrm{g}}$ is sensitive to the regions of $v(\mathrm{~T})$ that are used for linear fitting. As recommended in the literature [20], we used regions of $v(\mathrm{~T})$ data where the CVTE is stable and that are far from the transition region ( $\leq 180 \mathrm{~K}$ and $\geq 675 \mathrm{~K}$, as indicated by dashed vertical lines in Fig. $2 b$ ). The simulated glass transition temperatures of pDCPD-3 and pDCPD- 6 are $448 \pm 5 \mathrm{~K}$ and $462 \pm 3 \mathrm{~K}$, respectively. The similarity of these two values is reasonable given that the molecular weight between cross-links is not that different; in a previous simulation study of epoxy thermosets, doubling $\mathrm{M}_{\mathrm{C}}$ resulted in only a modest change in $T_{g}$ [37]. Interestingly, no inflection point is evident for pDCPD-1, indicating that no glass transition occurred on the timescale of these simulations, even at very high temperatures. We presume that the high extent of cross-linking in pDCPD-1 created a highly rigid network structure, thereby eliminating the large-scale segmental motions associated with the glass transition.

In a recent experimental study, Knorr et al. found that cross-linked pDCPD with $\mathrm{M}_{\mathrm{C}}=670 \mathrm{~g} / \mathrm{mol}$, which is intermediate between pDCPD-3 and pDCPD-6, has a glass transition temperature of $415 \mathrm{~K}$ [8]. The disparity of about $40 \mathrm{~K}$ between the simulated and experimental $\mathrm{T}_{\mathrm{g}}$ values is attributable to the vast difference in cooling rate between simulated and experimental systems (i.e., $\sim 11$ orders of magnitude faster in simulations). One method to correct for the difference in cooling rate is to assume an increase in $\mathrm{T}_{\mathrm{g}}$ of roughly 3-5 $\mathrm{K}$ for every order of magnitude increase in cooling rate [38], which yields a reasonable estimate for the shift in $\mathrm{T}_{\mathrm{g}}(33-55 \mathrm{~K})$. Considering the difference in cooling rate between experiment and simulation, the estimates of simulated $\mathrm{T}_{\mathrm{g}}$ are reasonable.

Next we discuss the effect of $\mathrm{M}_{\mathrm{C}}$ on the thermal and volumetric properties of pDCPD. In the rubbery region, increasing $\mathrm{M}_{\mathrm{C}}$ (i.e., going from pDCPD-1 to pDCPD-3 to pDCPD-6) increases both the specific volume and the CVTE (Fig. 2), which is a typical trend with $\mathrm{M}_{\mathrm{C}}[2,21,37]$. In the glassy region, CVTE is not strongly affected by $\mathrm{M}_{\mathrm{C}}$, because at low temperature chain entropy is dominated by intermolecular interactions, which are largely independent of chain connectivity. However, specific volume increases slightly with decreasing $\mathrm{M}_{\mathrm{C}}$ in the glassy region. This result can be explained by a reduction in packing efficiency near the relatively rigid pDCPD cross-links, as rigid cross-links are suggested to reduce packing efficiency in their vicinity [14]. Additionally, as $\mathbf{M}_{\mathrm{C}}$ decreases the linear segments become more rigid, which likely decreases the packing efficiency by limiting the configurations available to the chains. 
Table 2. Comparison of thermal and volumetric properties of pDCPD in simulations and experiments.

\begin{tabular}{|c|c|c|c|c|c|c|}
\hline Polymer network & $\begin{array}{c}\text { Specific } \\
\text { volume, } 295 \mathrm{~K} \\
\left(\mathrm{~cm}^{3} / \mathrm{g}\right) \\
\end{array}$ & $\begin{array}{c}\text { Specific } \\
\text { volume, } 465 \mathrm{~K} \\
\left(\mathrm{~cm}^{3} / \mathrm{g}\right) \\
\end{array}$ & $\begin{array}{c}\text { CVTE, } \\
\text { glassy } \\
\mu \mathrm{m} /(\mathbf{m} \cdot \mathbf{K}) \\
\end{array}$ & $\begin{array}{c}\text { CVTE, } \\
\text { rubbery } \\
\mu \mathrm{m} /(\mathbf{m} \cdot \mathbf{K}) \\
\end{array}$ & $\begin{array}{c}\mathbf{T}_{\mathbf{g}} \\
(\mathbf{K})\end{array}$ & $\underset{(\mathrm{g} / \mathrm{mol})}{\mathbf{M}_{\mathrm{C}}^{\mathrm{a}}}$ \\
\hline pDCPD-1 (sim.) & $0.996 \pm 0.005$ & $1.012 \pm 0.006$ & $83 \pm 3$ & $84 \pm 16$ & nd & 198 \\
\hline pDCPD-3 (sim.) & $0.989 \pm 0.001$ & $1.031 \pm 0.001$ & $179 \pm 5$ & $384 \pm 32$ & $448 \pm 5$ & 463 \\
\hline pDCPD-6 (sim.) & $0.983 \pm 0.001$ & $1.031 \pm 0.001$ & $194 \pm 4$ & $514 \pm 24$ & $462 \pm 3$ & 859 \\
\hline pDCPD (expt. [8]) & $0.961 \pm 0.003$ & $1.020 \pm 0.021$ & $258 \pm 3$ & $603 \pm 18$ & $415 \pm 9$ & $670 \pm 50$ \\
\hline
\end{tabular}

\section{B. Molecular mechanisms of the glass transition}

The glass transition occurs when the timescales of molecular relaxation and of observation are comparable [39]. A simple way to examine relaxations is through the motions of individual atoms [40], which we quantify by calculating the root-mean-square fluctuation (RMSF) of each atom over a period of time:

$$
R M S F_{i}=\sqrt{\frac{1}{T} \sum_{t=1}^{T}\left[r_{i}(t)-\bar{r}_{i}\right]^{2}}
$$

where $T$ is the number of snapshots over the time period, $r_{i}(t)$ is the position of atom $i$ in each snapshot $t$, and $\bar{r}_{i}$ is the average position of the atom during the time period. Larger values of RMSF indicate greater atomic mobility. First, we consider the effects of $\mathrm{M}_{\mathrm{C}}$ and the length of the time period $T$ (Fig. 3a). For 
pDCPD-3 and pDCPD-6, increasing the time period increases the average RMSF, whereas for pDCPD-1 the time period has no effect on the average RMSF. This result suggests that atoms in pDCPD-1 are confined to small spatial regions; they are essentially frozen in place relative to the network structure, even at high temperature. Below $\mathrm{T}_{\mathrm{g}}$, the atomic mobility is similar regardless of $\mathrm{M}_{\mathrm{C}}$, because the behavior is dominated by non-covalent interactions rather than by chain mobility. In all three networks, increasing temperature increases average atomic mobility, and the trend is similar to that of the specific volume (Fig. 2a): in pDCPD-3 and pDCPD-6 the atomic mobility increases at a greater rate above $\mathrm{T}_{\mathrm{g}}$, while in pDCPD1 mobility increases at a constant slow rate.

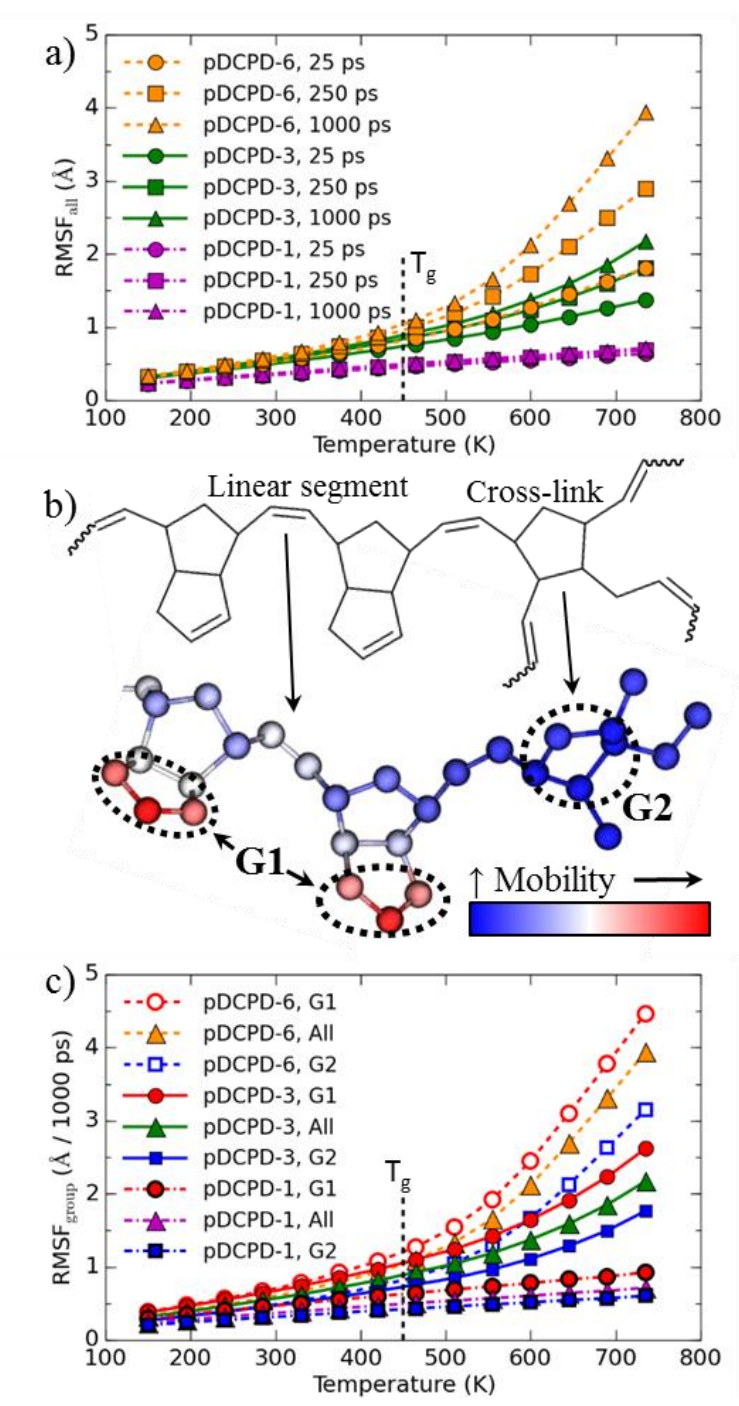

Figure 3. Atomic mobility through the glass transition. a) Average RMSF of all carbon atoms for pDCPD-1, pDCPD-3, pDCPD-6 over 25, 250, and 1000 ps as a function of temperature. The approximate location of $\mathrm{T}_{\mathrm{g}}$ is shown. b) Diagram of atomic mobility in various atoms in PDCPD networks and definition of two chemical groups, cyclopentenic rings in linear segments (G1) and cross-links (G2). For clarity, only carbon atoms are shown. c) 
Comparison of the average RMSF to the RMSF of the chemical groups G1 and G2. Error bars are smaller than the symbol size.

Heterogeneous mobility is associated with the glass transition in cross-linked epoxy [40]. To examine heterogeneity in atomic mobility, we calculate the average RMSF for each chemically-unique atom within the pDCPD network structure. Some atoms have greater mobility than others, as shown qualitatively in Fig. 3b. In particular, atoms at the tip of the cyclopentenic rings in the linear segments (labeled G1) have the highest mobility, while atoms in the cross-link cyclopentanic rings (labeled G2) have the lowest mobility. We quantify this difference in Fig. 3c, which shows the mobility of these atom groups relative to the average: regardless of $\mathrm{M}_{\mathrm{C}}$, the linear segments are more mobile, and cross-links are less mobile. This is a reasonable result, since the cross-links are constrained in multiple opposing directions and, likewise, the linear segments are less constrained. These results are unsurprising given the success of spatially-constrained cross-links in theoretical models of rubber elasticity [41].

Although the atomic mobility demonstrates small-scale relaxations, the glass transition is associated with large-scale relaxations of polymer segments (i.e., the $\alpha$-relaxation). To characterize the timescale of segmental relaxation, we calculate the autocorrelation function of the end-to-end vectors, $\vec{R}_{E E}$, of linear segments in pDCPD-6 (Fig. 4a). The autocorrelation function, $C(t)$, shows the relaxation rate of linear segments and is defined as:

$$
C(t)=\frac{\left\langle\vec{R}_{E E}\left(t_{0}\right) \cdot \vec{R}_{E E}\left(t_{0}+t\right)\right\rangle}{\left\langle R_{E E}^{2}\right\rangle}
$$

where $R_{E E}$ is the end-to-end distance, $t_{0}$ is the initial time, $t$ is the current time, and angle brackets indicate ensemble averaging. At low temperatures, segmental relaxation occurs slowly, while increasing the temperature accelerates relaxation (Fig. 4b). To quantify the effect of temperature, we fit $C(t)$ to a stretched exponential function:

$$
C(t)=C_{0} e^{-\left(\frac{t}{\tau}\right)^{\beta_{K W W}}}
$$

where $C_{0} \approx 1.0$ is a fitting coefficient, $\tau$ is a time constant, and $\beta_{K W W}$ is the Kohlrausch-Williams-Watts stretching exponent [42]. Stretched exponential functions are commonly used to describe the broad relaxation times associated with the glass transition [43], and Eq. (5) fits our data well (dashed lines in Fig. 4b). The characteristic relaxation time $\tau_{C}$ of linear segments is then given by:

$$
\tau_{C}=\int_{0}^{\infty} C(t) d t=C_{0} \frac{\tau}{\beta_{K W W}} \Gamma\left(\frac{1}{\beta_{K W W}}\right)
$$

where $\Gamma$ is the gamma function, and the characteristic rate of segmental relaxation is taken as $\tau_{C}^{-1}$. The characteristic rate decreases approximately linearly with inverse temperature over the entire temperature 
range, 90 to $885 \mathrm{~K}$. We model the temperature dependence of the end-to-end relaxation using the Arrhenius equation:

$$
\ln \left(\tau_{C}^{-1}\right)=\ln (A)-\frac{E_{a}}{R}\left(\frac{1}{T}\right)
$$

where $A$ is the frequency pre-factor, $E_{a}$ is the activation energy, and $R$ is the gas constant. We determine $E_{a}$ by fitting Eq. (7) to the temperature-dependent relaxation rates, which yields an activation energy of $E_{a}=697 \pm 155 \mathrm{~kJ} / \mathrm{mol}$. This value of $E_{a}$ is consistent with typical activation energies for $\alpha$-relaxations (e.g., $~ 160-800 \mathrm{~kJ} / \mathrm{mol}$ in cross-linked networks $[9,44,45]$ ), suggesting that these segment-scale motions are indeed a major factor in the glass transition of pDCPD.

a)
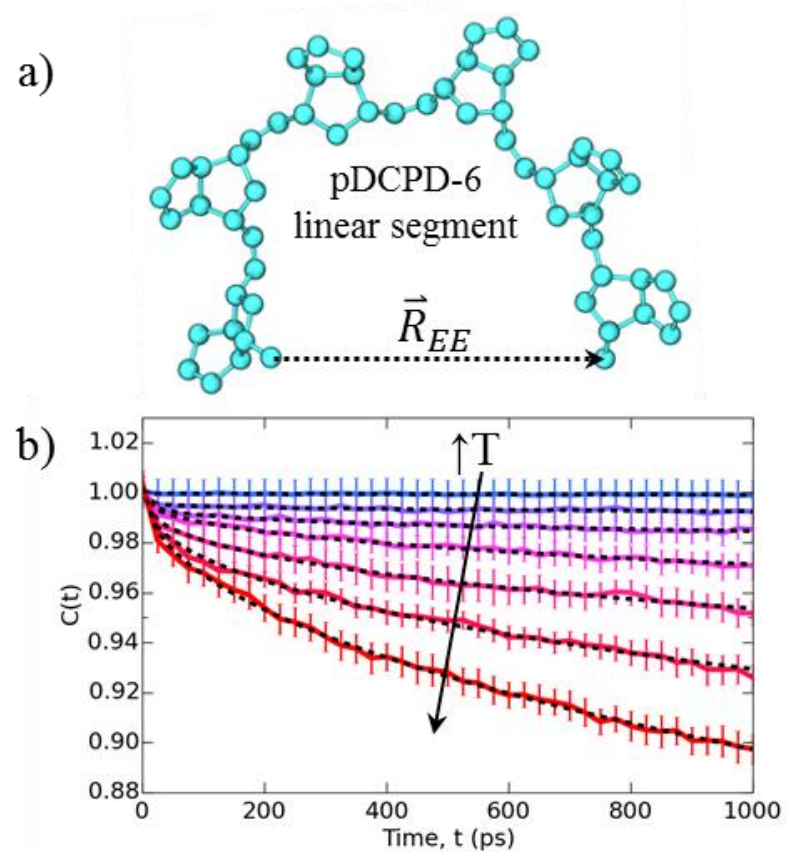

Figure 4. Segment-scale relaxation. a) Definition of end-to-end vector $\left(\mathrm{R}_{\mathrm{EE}}\right)$ used to describe segmental relaxation. b) Autocorrelation of the end-to-end vector as a function of temperature. Dashed black lines are fits to a stretched exponential function, Eq. (5). Error bars are the standard error of the mean of five replicas. Results are for pDCPD6. 

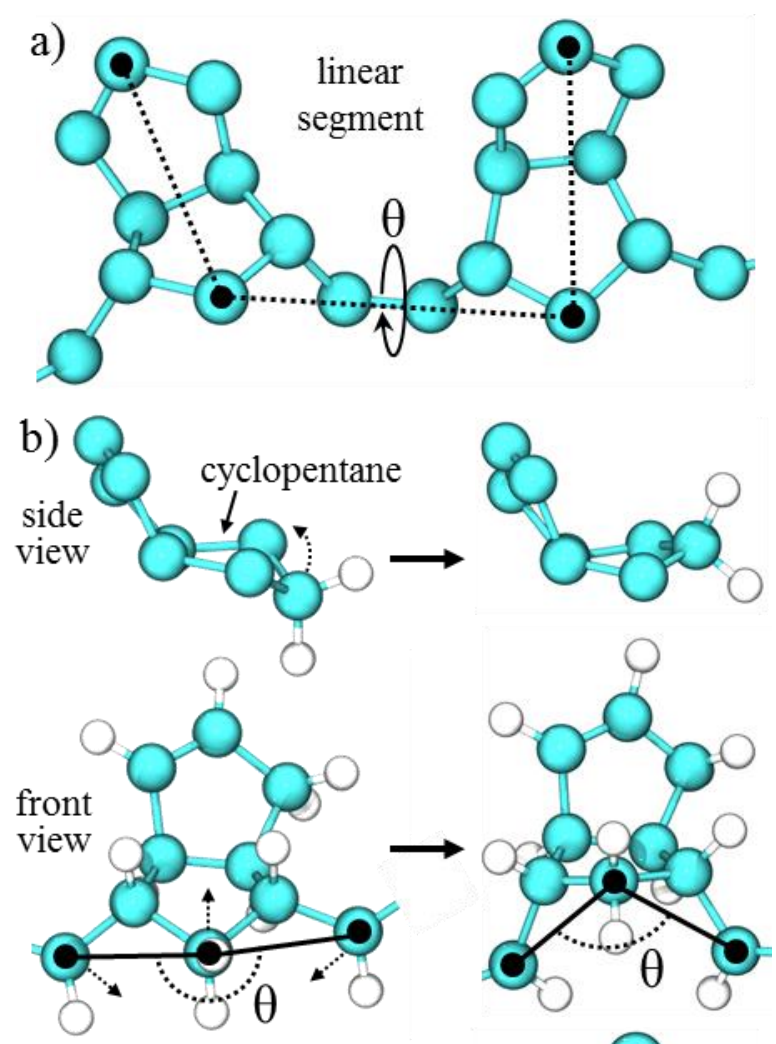

c)

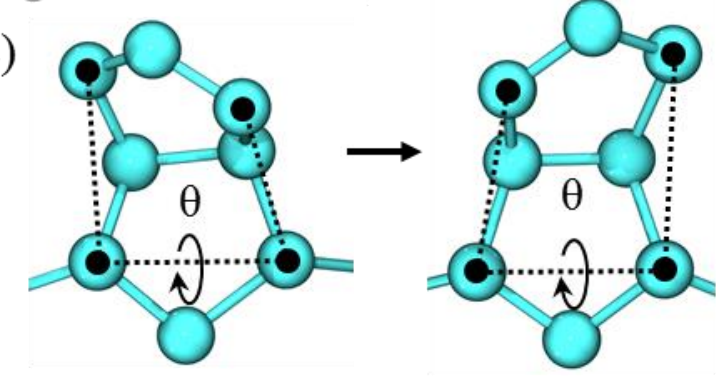

d)

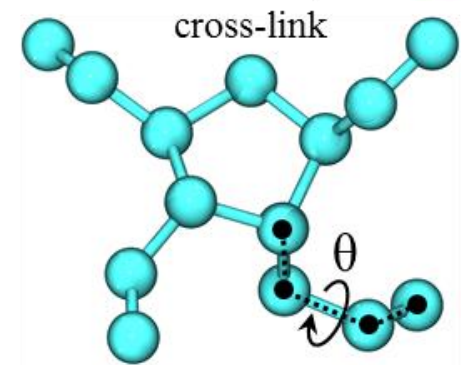

Figure 5. Diagrams of sub-segmental relaxations. For clarity, not all atoms are shown. a) Torsion angle used to describe twisting between neighboring DCPD monomers. Only the two central monomers in pDCPD-6 linear segments were included. b) (top) Diagram of cyclopentane ring flip in linear segment of pDCPD. (bottom) Definition of angle that describes the concerted cyclopentane ring flip and local motion of the network backbone. c) Torsion angle used to describe the double ring twisting motion in linear segments. d) Torsion angle used to describe motion of long, relatively flexible linkage between cross-links and linear segments. 
Sub-segmental molecular motions that occur below $\mathrm{T}_{\mathrm{g}}$ can contribute to mechanical properties and have been reported to influence ballistic performance $[9,10]$. Thus, the incorporation of specific molecular motions with known characteristics can be used to tune material properties. Knorr et al. used dielectric spectroscopy to identify two sub- $\mathrm{T}_{\mathrm{g}}$ transitions that were associated with molecular motions in pDCPD and reported their activation energies [8]. The $\beta$-transition $\left(E_{a, \beta}=76 \mathrm{~kJ} / \mathrm{mol}\right)$ was suggested to be associated with ring motions, and the $\delta$-transition $\left(E_{a, \delta}=32 \mathrm{~kJ} / \mathrm{mol}\right)$ was suggested to be associated with linkages between DCPD monomers. We note that these activation energies are typical of sub- $\mathrm{T}_{\mathrm{g}}$ relaxations $(10-80 \mathrm{~kJ} / \mathrm{mol})$ [9]. We now quantify several sub-segmental motions for the purpose of identifying specific molecular motions and associating these with sub- $\mathrm{T}_{\mathrm{g}}$ transitions in $\mathrm{pDCPD}$.

We first examine a motion that is related to the greater mobility of the cyclopentenic rings in the linear segments. We describe a twisting motion between neighboring DCPD monomers in linear segments using a torsion angle (Fig. 5a). To quantify this twisting motion, we calculate the autocorrelation function of the torsion angle, $\theta$, which is defined as:

$$
C(t)=\left\langle\cos \left[\theta\left(t_{0}\right)-\theta\left(t_{0}+t\right)\right]\right\rangle
$$

where $t_{0}$ is the initial time, $t$ is the current time, and angle brackets indicate ensemble averaging. We perform the Arrhenius analysis using Eqs. (5-8), yielding an activation energy of $E_{a}=225 \pm 12 \mathrm{~kJ} / \mathrm{mol}$. This value is larger than typical sub- $\mathrm{T}_{\mathrm{g}}$ activation energies, suggesting that the twisting motion is associated with the $\alpha$-transition.

In some common epoxy networks, the $\beta$-relaxation is associated with ring flip motions [11], thus we examine the motions of the cyclopentanic rings in linear pDCPD segments. To alleviate ring strain, cyclopentane rings assume non-planar conformations with one or two carbon atoms out-of-plane from the other atoms [46]. The out-of-plane atom(s) can flip between sides of the ring plane, forcing the substituents of those atoms to swap between axial and equatorial positions (Fig. 5b, top). In linear pDCPD, these ring flip motions lead to a concerted motion of the cyclopentanic group and other carbon atoms in the network backbone, which is conveniently described by an angle between three atoms (Fig. $5 \mathrm{~b}$, bottom). The Arrhenius analysis yields an activation energy of $E_{a}=166 \pm 16 \mathrm{~kJ} / \mathrm{mol}$. This barrier is larger than typical sub- $\mathrm{T}_{\mathrm{g}}$ relaxations, suggesting that the ring flip and concomitant backbone rearrangement are associated with the $\alpha$-relaxation. We speculate that the intra-chain twisting (Fig. 5a) and ring flip (Fig. 5b) motions may facilitate the inter-chain motions of the $\alpha$-relaxation by rearranging the segment backbone.

Another ring-related motion occurs in linear pDCPD segments when the cyclopentenic and cyclopentanic rings twist relative to each other (Fig. 5c). We define a torsion angle that qualitatively correlates with the twisting motion and conduct the Arrhenius analysis, which yields an activation energy 

experimental value $E_{a, \delta}=32 \mathrm{~kJ} / \mathrm{mol}$ in $\mathrm{pDCPD}$, suggesting that this twisting motion is associated with the $\delta$-relaxation in pDCPD.

Knorr et al. suggested that the $\delta$-relaxation in pDCPD may be associated with the short linkages between cross-links and linear segments [8]. In each cross-link, one of these linkages is one carbon longer and more flexible than the others (Fig. 1c, Fig. 5d). This linkage resembles a single repeat unit of linear polybutadiene, which has a $\beta$-relaxation with $E_{a}=42 \mathrm{~kJ} / \mathrm{mol}$ [47]. We characterize the crankshaft motion of this linkage by defining a torsion angle around the central bond (Fig. 5d) and conducting the Arrhenius analysis, yielding $E_{a}=67 \pm 5 \mathrm{~kJ} / \mathrm{mol}$. This value is larger than the $\beta$-relaxation in polybutadiene and the experimental $E_{a, \delta}$, but it is similar to the experimental value $E_{a, \beta}=76 \mathrm{~kJ} / \mathrm{mol}$ in $\mathrm{pDCPD}$, suggesting that this motion is instead associated with the $\beta$-relaxation in pDCPD. This relaxation does not occur at the other linkages between pDCPD monomers, because the torsion across the double bond in these linkages does not significantly relax at any temperature, as this would require a cis-trans isomerization.

\section{Conclusion}

We conducted atomistic molecular dynamics simulations of cross-linked poly(dicyclopentadiene) (pDCPD) networks with the goal of characterizing the glass transition behavior. We developed three model pDCPD systems with differing molecular weights between cross-linking junctions. The simulated networks were annealed through the glass transition. The thermal and volumetric properties were assessed (i.e., specific volume, coefficient of thermal expansion, glass transition temperature) and found to be in reasonable agreement with experimental values, although the $\mathrm{T}_{\mathrm{g}}$ and coefficient of thermal expansion were higher and lower than experiments, respectively. These discrepancies are common in simulations of polymer networks, where the increased $\mathrm{T}_{\mathrm{g}}$ is typically attributed to the high simulated cooling rate, and the lowered volumetric expansion to the ideal topology of the simulated networks.

The molecular mechanism of the glass transition was assessed by measuring the mobility of atoms across the glass transition. Atoms belonging to cross-linkers have only modest mobility above $\mathrm{T}_{\mathrm{g}}$, and are immobile below $\mathrm{T}_{\mathrm{g}}$ compared to other atoms in the network. Conversely, the atoms in linear segments are highly mobile above the glass transition but are reduced to essentially the same low mobility as other atoms at temperatures below $\mathrm{T}_{\mathrm{g}}$. If all of these highly mobile atoms are constrained by crosslinking reactions, as in one of our systems (pDCPD-1), the glass transition is completely removed.

We also examined molecular motions at various length scales. At the highest scale, we quantified the relaxations of linear pDCPD segments, finding an activation energy $(\sim 700 \mathrm{~kJ} / \mathrm{mol})$ that is consistent with typical $\alpha$-relaxations. At smaller scales, we identified an inter-monomer twisting motion, and we identified an intra-monomer motion involving a concerted cyclopentane ring flip and local rearrangement 
of the network backbone. These two motions, due to their large activation energies ( $200 \mathrm{~kJ} / \mathrm{mol})$, also appear to be associated with the glass transition. These motions may be intra-chain motions that facilitate the concerted inter-chain segmental motions of the $\alpha$-relaxation. At the smallest scale, we identified a crankshaft motion in a linkage between cross-links and linear segments, which has an activation energy $(\sim 70 \mathrm{~kJ} / \mathrm{mol})$ consistent with typical sub- $\mathrm{T}_{\mathrm{g}}$ relaxations and with the experimental $\beta$-transition. Finally, we identified a ring twisting motion in the double-ring hexahydropentalene units in linear pDCPD with an activation energy $(\sim 40 \mathrm{~kJ} / \mathrm{mol})$ characteristic of sub-T $\mathrm{T}_{\mathrm{g}}$ relaxations and similar to the experimental $\delta$ transition.

A recent study by Knorr et al. demonstrated that pDCPD cross-linked polymers can overcome what is often a trade-off of mechanical properties between strength and toughness [8]. Specifically, crosslinked pDCPD was found to have exceptional ballistic performance compared to epoxy resins, which appears to be related to the high fracture toughness of pDCPD. The molecular mechanism underlying this exceptional toughness was not readily identifiable; however, pDCPD contains numerous bulky sidechains that may improve ductility by reducing packing efficiency. Building upon the present study where we investigated the thermal properties of $\mathrm{pDCPD}$, in future work we will investigate the role of side chains and other local network structures on the mechanical properties of pDCPD networks.

\section{Acknowledgements}

RME was supported by an appointment to the Postgraduate Research Participation Program at the U.S. Army Research Laboratory administered by the Oak Ridge Institute for Science and Education through an interagency agreement between the U.S. Department of Energy and USARL. This work was supported by grants of computer time from the DOD High Performance Computing Modernization Program at the U.S. Air Force Research Laboratory and U.S. Army Engineer Research and Development Center DoD Supercomputing Resource Centers. The authors thank Dr. Kevin Masser (USARL) and Dr. Daniel Knorr (USARL) for useful discussions. 


\section{References}

[1] D.B. Knorr Jr, J.H. Yu, A.D. Richardson, M.D. Hindenlang, I.M. McAninch, J.J. La Scala, J.L. Lenhart, Polymer 53 (2012) 5917.

[2] L.M. McGrath, R.S. Parnas, S.H. King, J.L. Schroeder, D.A. Fischer, J.L. Lenhart, Polymer 49 (2008) 999.

[3] H.S. Kaufman, J. Appl. Polym. Sci. 14 (1970) 253.

[4] M. Aldridge, C. Shankar, Changgua Zhen, Lang Sui, J. Kieffer, M. Caruso, J. Moore, J. Compos. Mater. 44 (2010) 2605.

[5] Z.I. Kalcioglu, R.A. Mrozek, R. Mahmoodian, M.R. VanLandingham, J.L. Lenhart, K.J. Van Vliet, J. Biomech. 46 (2013) 1583.

[6] R.A. Mrozek, P.J. Cole, K.J. Otim, K.R. Shull, J.L. Lenhart, Polymer 52 (2011) 3422.

[7] N.K. Nielsen, R.F. Landel, Mechanical properties of polymers and composites, Marcel Dekker, New York, 1994.

[8] D.B. Knorr Jr, K.A. Masser, R.M. Elder, T.W. Sirk, M.D. Hindenlang, J.H. Yu, A.D. Richardson, S.E. Boyd, W.A. Spurgeon, J.L. Lenhart, Compos. Sci. Technol. 114 (2015) 17.

[9] E. Mark, James E., Physical Properties of Polymers Handbook, Springer Science \& Business Media, New York, 2007.

[10] P.I. Vincent, Polymer 15 (1974) 111.

[11] J.F. Shi, P.T. Inglefield, A.A. Jones, M.D. Meadows, Macromolecules 29 (1996) 605.

[12] S. Maiti, C. Shankar, P.H. Geubelle, J. Kieffer, J. Eng. Mater. Technol. 128 (2006) 595.

[13] C. Shankar, Ph.D. thesis, University of Michigan, 2009.

[14] C.L. Soles, F.T. Chang, B.A. Bolan, H.A. Hristov, D.W. Gidley, A.F. Yee, J. Polym. Sci., Part B: Polym. Phys. 36 (1998) 3035.

[15] E. Crawford, A.J. Lesser, J. Polym. Sci., Part B: Polym. Phys. 36 (1998) 1371.

[16] D. Schaubroeck, S. Brughmans, C. Vercaemst, J. Schaubroeck, F. Verpoort, J. Mol. Catal. A: Chem. 254 (2006) 180.

[17] G. Yang, J.K. Lee, Ind. Eng. Chem. Res. 53 (2014) 3001.

[18] T.A. Davidson, K.B. Wagener, D.B. Priddy, Macromolecules 29 (1996) 786.

[19] T.C. Mauldin, J.D. Rule, N.R. Sottos, S.R. White, J.S. Moore, J. R. Soc., Interface 4 (2007) 389.

[20] T.W. Sirk, K.S. Khare, M. Karim, J.L. Lenhart, J.W. Andzelm, G.B. McKenna, R. Khare, Polymer 54 (2013) 7048.

[21] T.W. Sirk, M. Karim, K.S. Khare, J.L. Lenhart, J.W. Andzelm, R. Khare, Polymer 58 (2015) 199.

[22] L.D. Landau, E.M. Lifshitz, Statistical Physics. Part 1: Course of Theoretical Physics, Pergamon Press Inc., Tarrytown, NY, 1980. 
[23] P.-H. Lin, R. Khare, Macromolecules 42 (2009) 4319.

[24] C.-W. Jang, T.W. Sirk, J.W. Andzelm, C.F. Abrams, Macromol. Theory Simul. 24 (2015) 260.

[25] D.M. Heyes, Phys. Rev. B: Solid State 49 (1994) 755.

[26] S. Panyukov, Y. Rabin, Phys. Rep. 269 (1996) 1.

[27] J. Wang, R.M. Wolf, J.W. Caldwell, P.A. Kollman, D.A. Case, J. Comp. Chem. 25 (2004) 1157.

[28] J. Wang, W. Wang, P.A. Kollman, D.A. Case, J. Mol. Graphics Modell. 25 (2006) 247.

[29] H. Sun, J. Phys. Chem. B 102 (1998) 7338.

[30] A. Jakalian, B.L. Bush, D.B. Jack, C.I. Bayly, J. Comp. Chem. 21 (2000) 132.

[31] A. Jakalian, D.B. Jack, C.I. Bayly, J. Comp. Chem. 23 (2002) 1623.

[32] R.W. Hockney, J.W. Eastwood, Computer simulation using particles, Institute of Physics Publishing, Philadelphia, 1988.

[33] W. Shinoda, M. Shiga, M. Mikami, Phys. Rev. B: Solid State 69 (2004) 134103.

[34] S. Plimpton, J. Comp. Phys. 117 (1995) 1.

[35] W. Humphrey, A. Dalke, K. Schulten, J. Mol. Graphics 14 (1996) 33.

[36] A.I. Jewett, Z. Zhuang, J.-E. Shea, Biophys. J. 104 (2013) 169a.

[37] N.J. Soni, P.-H. Lin, R. Khare, Polymer 53 (2012) 1015.

[38] M.D. Ediger, C.A. Angell, S.R. Nagel, J. Phys. Chem. 100 (1996) 13200.

[39] P.K. Gupta, J.C. Mauro, J. Chem. Phys. 126 (2007) 224504.

[40] P.-H. Lin, R. Khare, Macromolecules 43 (2010) 6505.

[41] P.J. Flory, Principles of Polymer Chemistry, Cornell University Press, Ithaca, New York, 1953.

[42] G. Williams, D.C. Watts, Trans. Faraday Soc. 66 (1970) 80.

[43] E. Vidal Russell, N.E. Israeloff, Nature 408 (2000) 695.

[44] R.E. Jensen, G.R. Palmese, S.H. McKnight, Dynamic Mechanical Analysis of E-Beam and Thermally Curable IPN Thermosets. Army Research Lab, DTIC accession number ADA400172, 2002.

[45] I.M. McAninch, Ph.D. thesis, Drexel University, 2014.

[46] E.V. Anslyn, D.A. Dougherty, Modern Physical Organic Chemistry, University Science Books, 2006.

[47] R. Casalini, K.L. Ngai, C.G. Robertson, C.M. Roland, J. Polym. Sci., Part B: Polym. Phys. 38 (2000) 1841. 
Figure 1

a)
DCPD

monomer
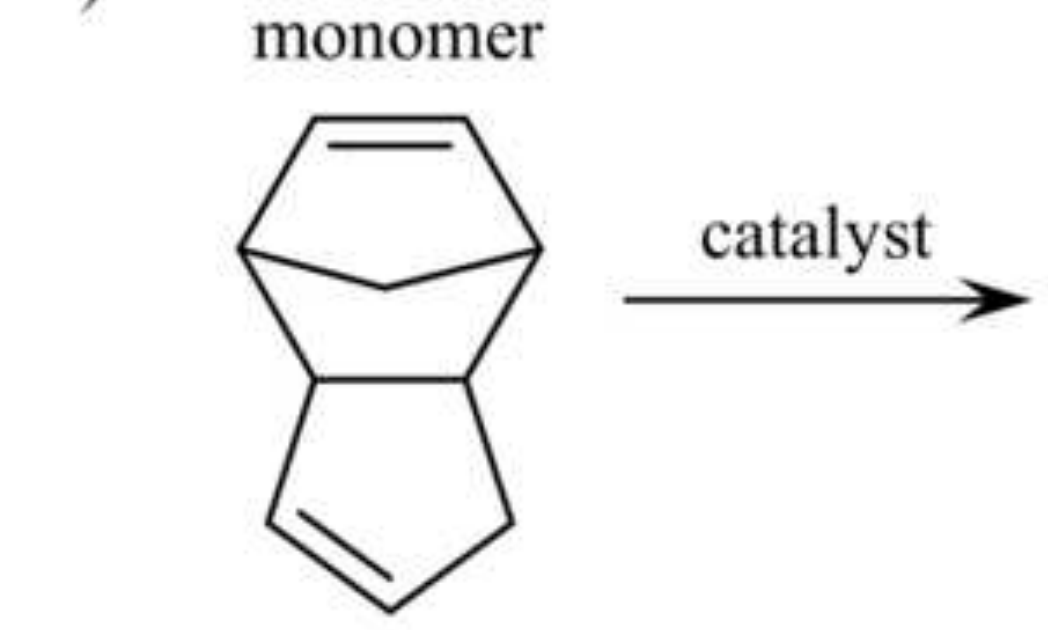

linear $\mathrm{pDCPD}$

छ
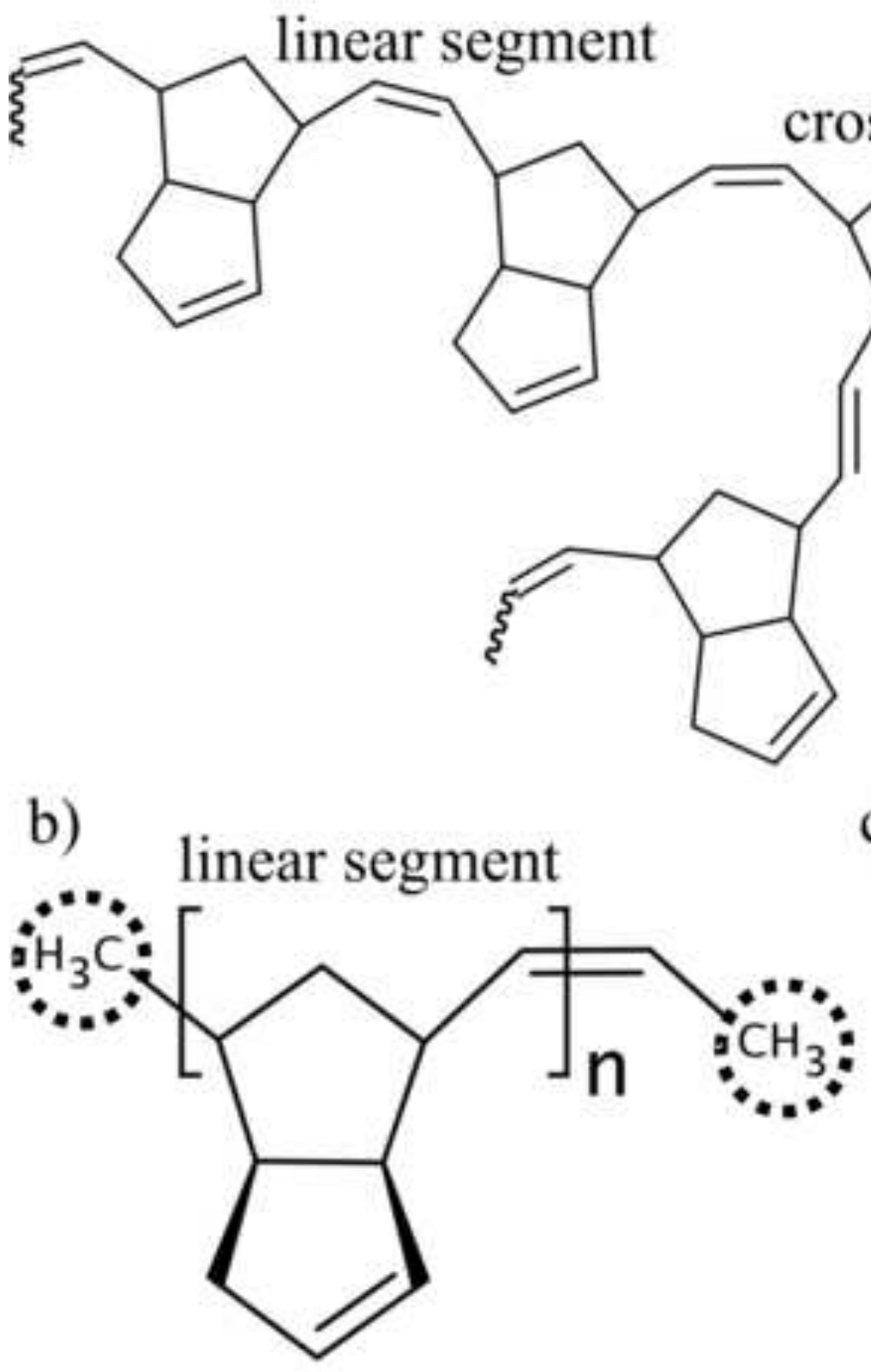

c)

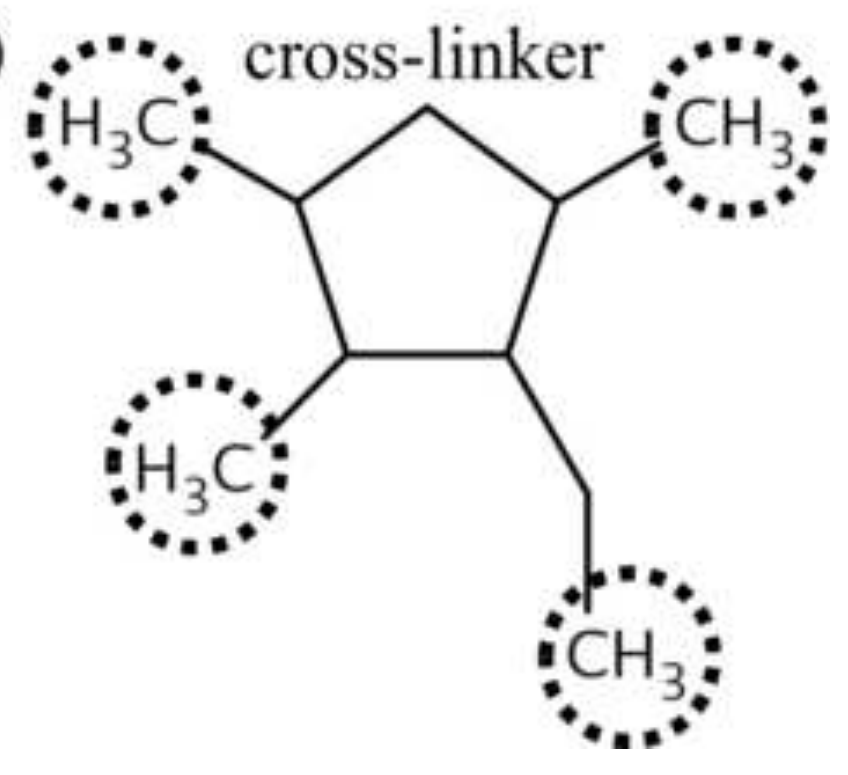


Figure 2
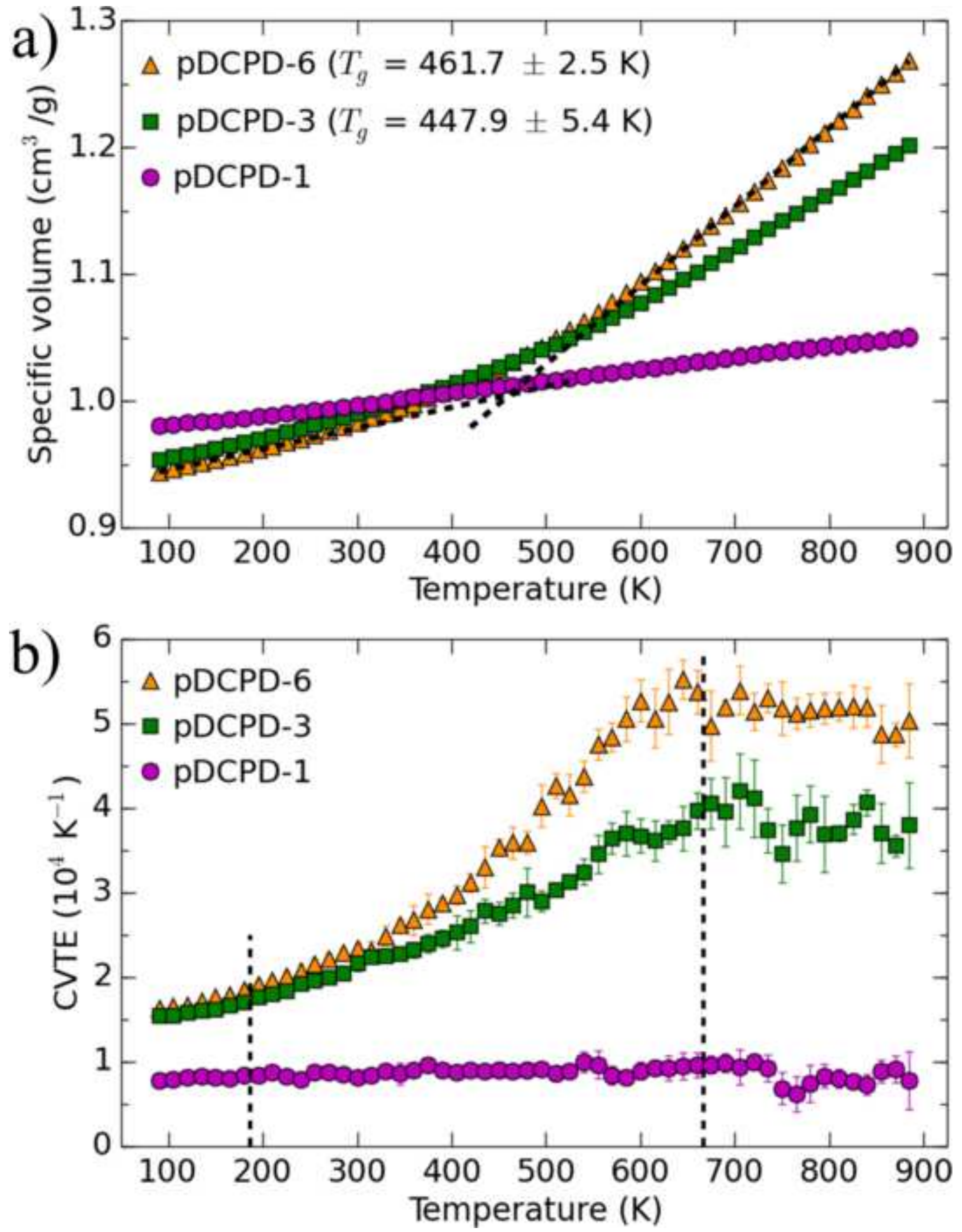
Figure 3

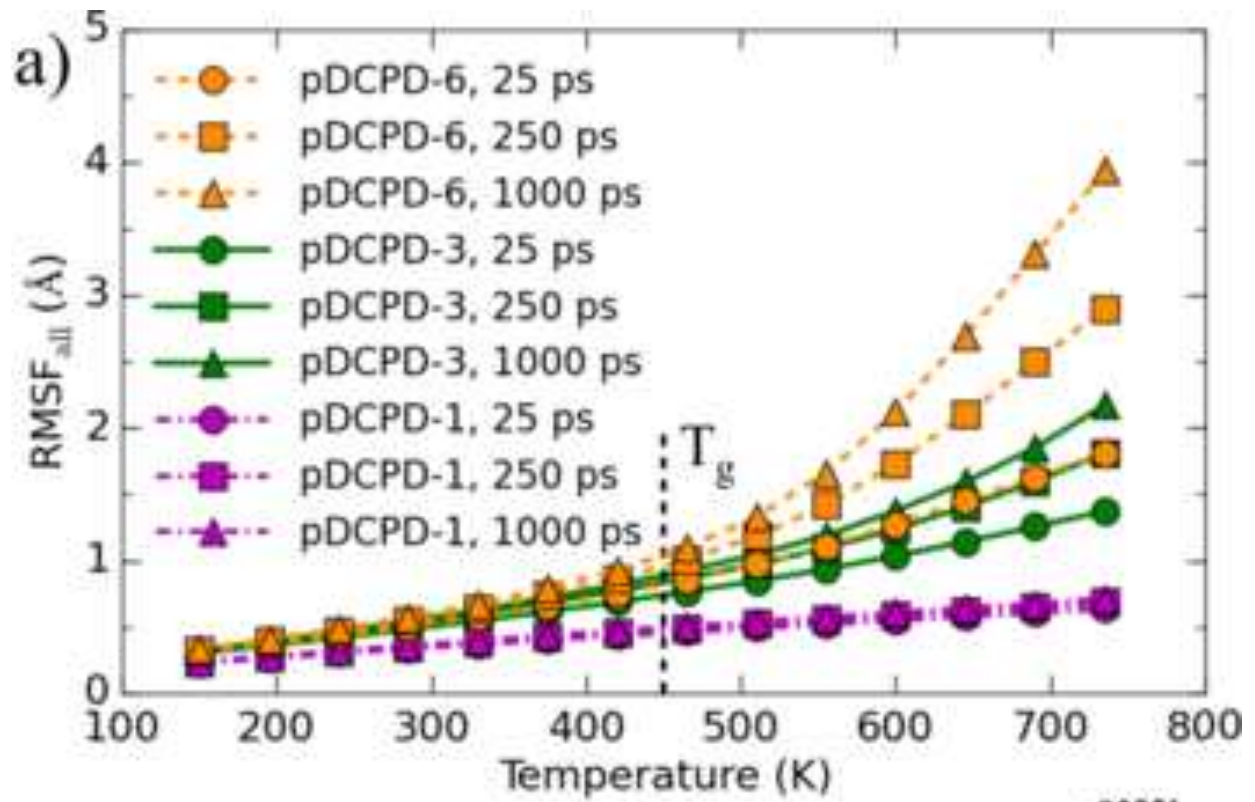

b)
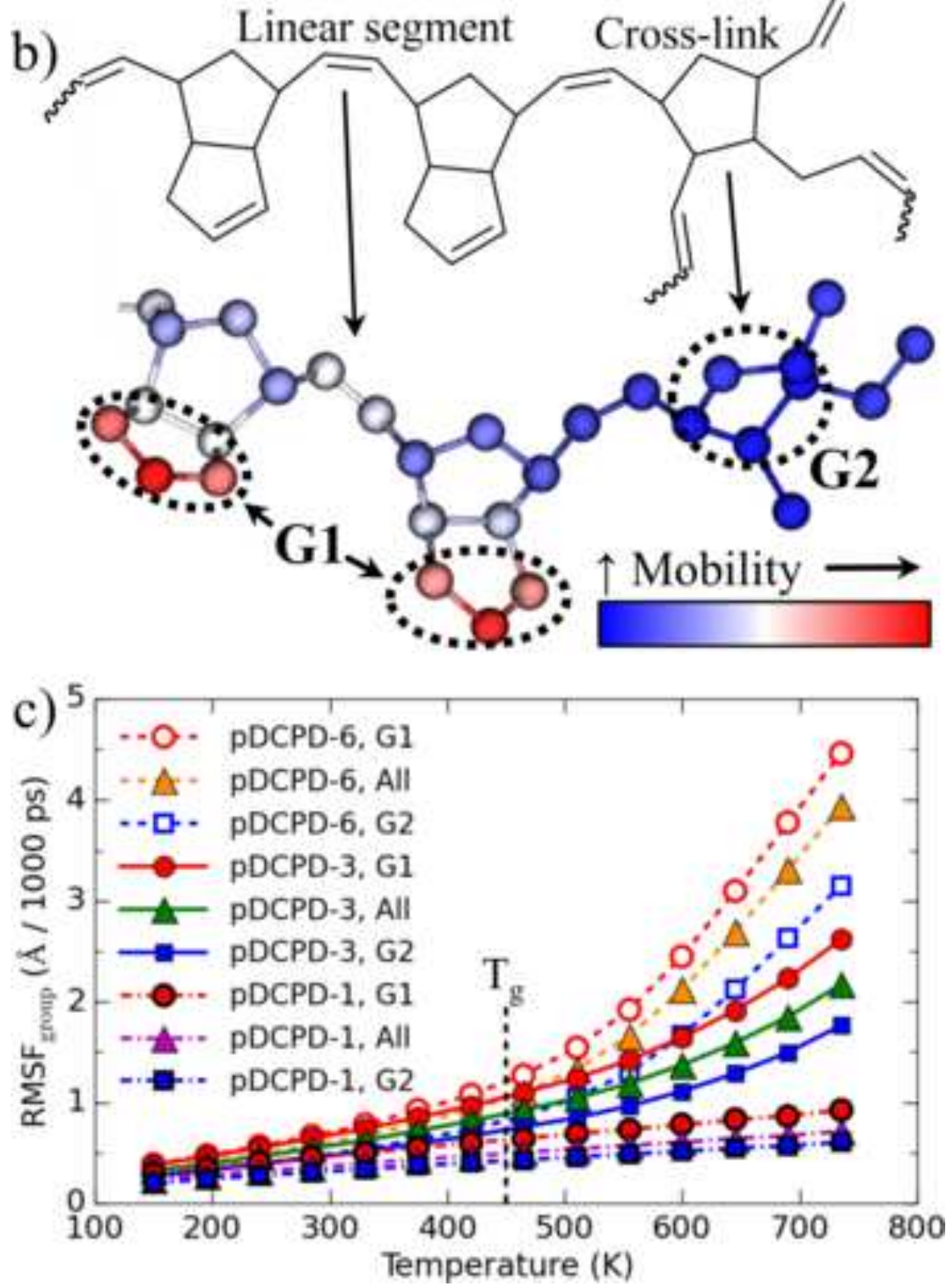
Figure 4

a)
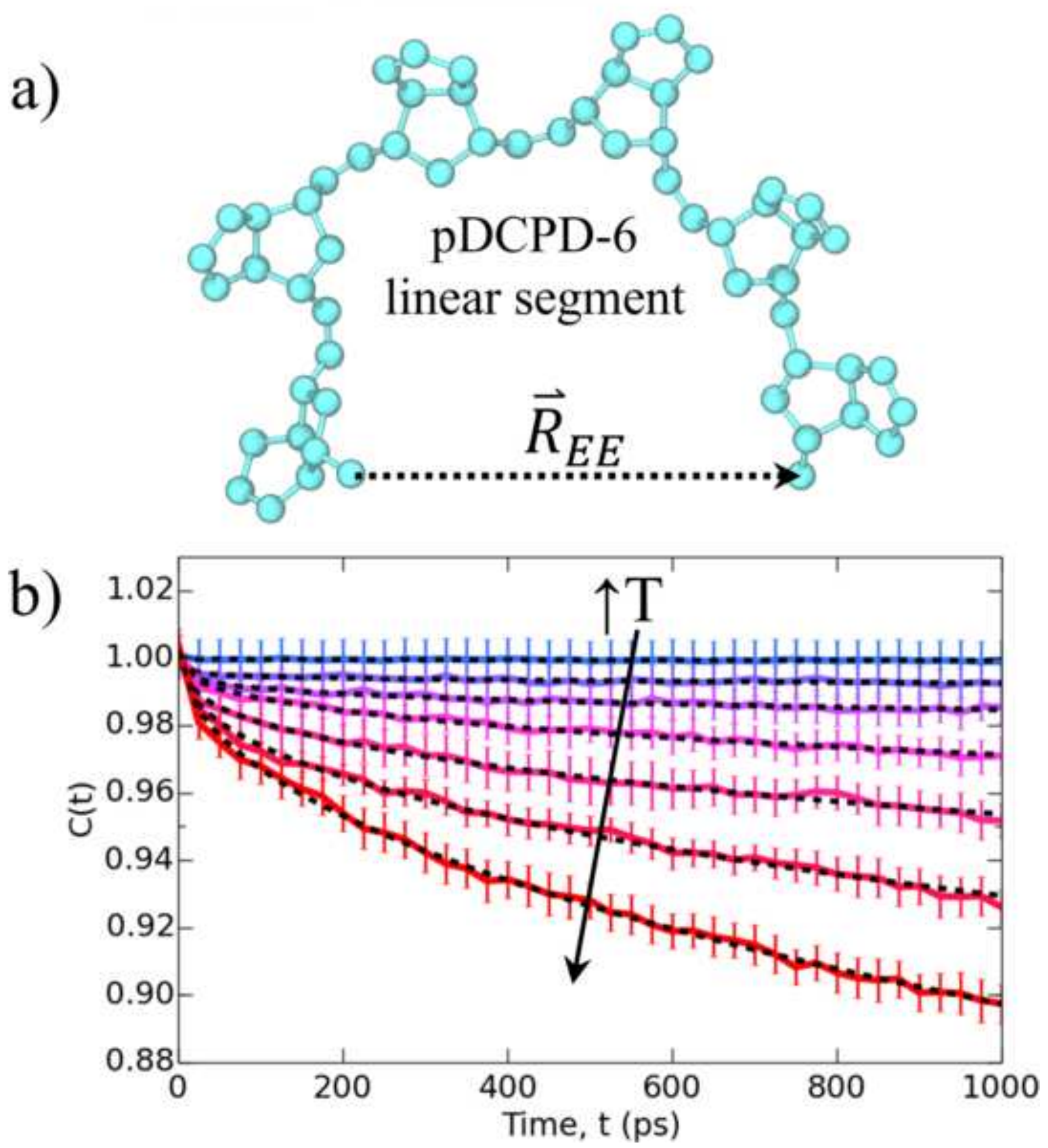
Figure 5
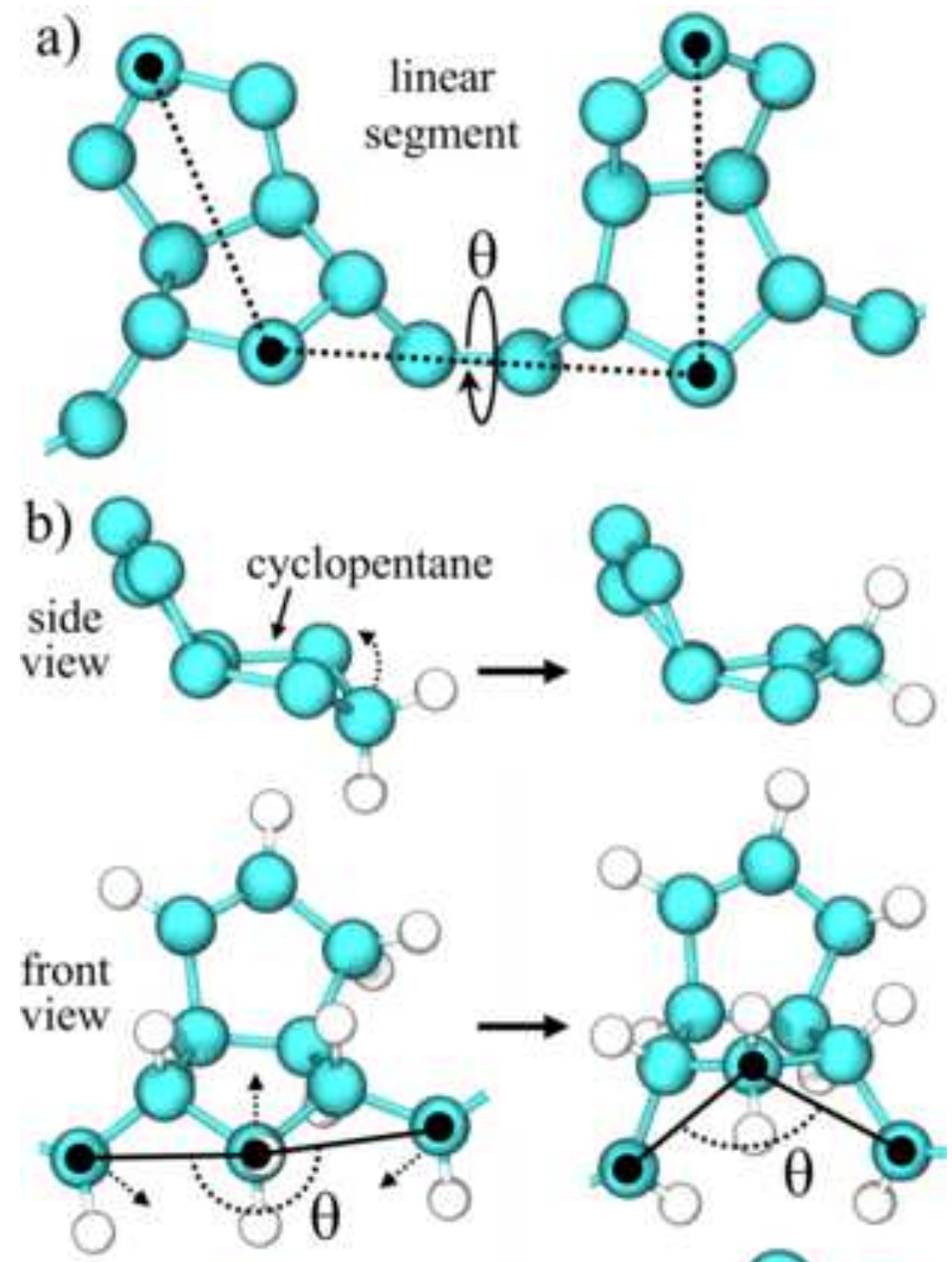

c)

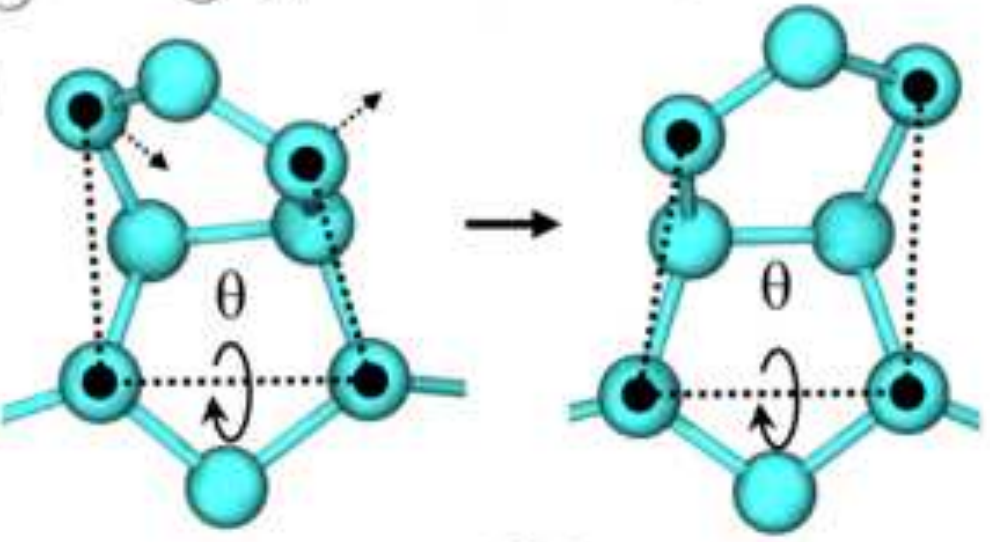

d)

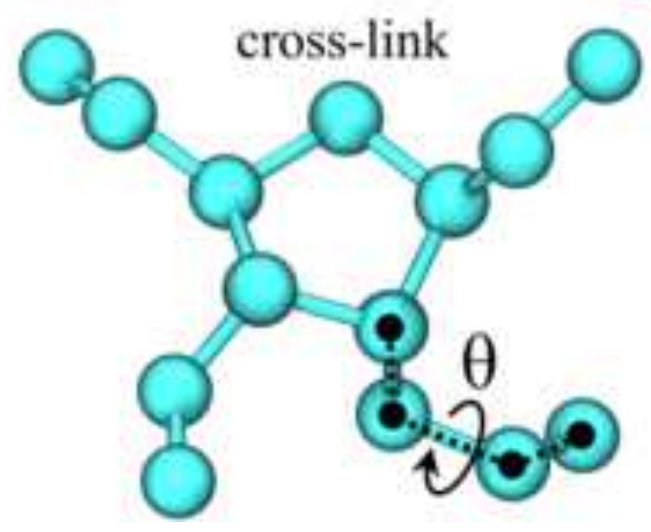

